\title{
Transcriptional Analysis of Flowering Time in Switchgrass
}

\author{
Carl-Erik Tornqvist ${ }^{1}$ (D) Brieanne Vaillancourt ${ }^{2}$. Jeongwoon Kim $^{2,3} \cdot$ C. Robin Buell ${ }^{2}$. \\ Shawn M. Kaeppler ${ }^{1} \cdot$ Michael D. Casler ${ }^{1,4}$
}

Published online: 27 April 2017

(C) The Author(s) 2017. This article is an open access publication

\begin{abstract}
Over the past two decades, switchgrass (Panicum virgatum) has emerged as a priority biofuel feedstock. The bulk of switchgrass biomass is in the vegetative portion of the plant; therefore, increasing the length of vegetative growth will lead to an increase in overall biomass yield. The goal of this study was to gain insight into the control of flowering time in switchgrass that would assist in development of cultivars with longer vegetative phases through delayed flowering. RNA sequencing was used to assess genome-wide expression profiles across a developmental series between switchgrass genotypes belonging to the two main ecotypes: upland, typically early flowering, and lowland, typically late flowering. Leaf blades and tissues enriched for the shoot apical meristem (SAM) were collected in a developmental series from emergence through anthesis for RNA extraction. RNA from samples that flanked the SAM transition stage was sequenced for expression analyses. The analyses revealed differential expression patterns between early- and late-flowering genotypes for known flowering time orthologs. Namely, genes shown to
\end{abstract}

Electronic supplementary material The online version of this article (doi:10.1007/s12155-017-9832-9) contains supplementary material, which is available to authorized users.

Carl-Erik Tornqvist

citornqvist@wisc.edu

1 Department of Agronomy and DOE Great Lakes Bioenergy Research Center, University of Wisconsin - Madison, Madison, WI 53706, USA

2 Department of Plant Biology and DOE Great Lakes Bioenergy Research Center, Michigan State University, East Lansing, MI 48824, USA

3 Present address: Monsanto Company, Chesterfield, MO 63017, USA

4 USDA Dairy Forage Research Center, Madison, WI 53706, USA play roles in photoperiod response and the circadian clock in other species were identified as potential candidates for regulating flowering time in the switchgrass genotypes analyzed. Based on their expression patterns, many of the differentially expressed genes could also be classified as putative promoters or repressors of flowering. The candidate genes presented here may be used to guide switchgrass improvement through marker-assisted breeding and/or transgenic or gene editing approaches.

Keywords Switchgrass $\cdot$ Biomass $\cdot$ Flowering time $\cdot$ RNAseq $\cdot$ Differential expression

\section{Introduction}

Switchgrass (Panicum virgatum) is a warm season, C4 perennial grass that is drought-tolerant and requires little fertilizer inputs to thrive [1]. A prairie grass native to the USA, with a wide adaptation range east of the Rocky Mountains, extending from the Gulf of Mexico to Canada, switchgrass has historically been used as a forage crop [2]. It was not until the early 1990s that the US Department of Energy (DOE) chose switchgrass as a model biofuel feedstock worthy of intensive research with goals for improvement [3].

There are two main ecotypes of switchgrass: lowland and upland. In general, lowland varieties are tall, have wide leaves with a bluish hue, grow upright, flower late, and are not coldtolerant. Uplands, on the other hand, are cold-hardy, earlyflowering, and, in general, have narrower leaves and smaller stature than lowlands. In switchgrass, as with many crops, later-flowering genotypes accumulate more biomass [4]. To make switchgrass cultivation an economically feasible endeavor for farmers as a biofuel feedstock, a minimum biomass yield must be achievable [4]. In the southern USA, there are 
many late-flowering lowland switchgrass varieties that yield high amounts of biomass to allow for efficient switchgrass cultivation [3]. In the North Central USA, however, the lowland varieties would not be reliable due to reduced winter survival. Therefore, the aim of this study was to identify flowering time gene candidates that could be employed to manipulate flowering time in upland, cold-tolerant cultivars.

From a developmental standpoint, the key event in determining flowering time is the transition of the shoot apical meristem (SAM) from the vegetative to reproductive phase. Several input pathways feed into determination of the flowering transition, with mechanisms that either promote or repress flowering. Ultimately, in the case of flowering activation, a signaling cascade results in gene expression and physiological changes in the SAM, transitioning it from a vegetative to a reproductive meristem. The signaling cascades converge on genes classified as floral pathway integrator (FPI) and floral meristem identity (FMI) genes [5], which act as hubs to sense inputs and to activate the meristem transition, respectively. Once the FMI genes are activated, the SAM begins the transition to reproductive development. This transition in switchgrass may occur several weeks before heading is observed, which is the exertion of the panicle from the surrounding leaves; heading date is a practical measure of flowering time. After the SAM has transitioned to reproductive growth, the remaining floral development is dependent, for the most part, on developmental processes running their course. Therefore, the SAM transition is the key molecular process defining flowering time.

The genetic pathway of flowering time has been studied extensively in model species, particularly the dicot Arabidopsis (Arabidopsis thaliana), in which many mechanisms have been largely elucidated $[5,6]$. The genetics of flowering time has also been studied intensely in monocots such as maize (Zea mays) (e.g., [7]), rice (Oryza sativa) (e.g., [5, 8]), and Brachypodium (Brachypodium distachyon) (e.g., $[5,9])$. Some aspects of the flowering time pathway in Arabidopsis are shared in monocots, as evidenced by the many orthologs of Arabidopsis genes found in such species [5].

The main flowering time input pathways are photoperiod, circadian clock, hormone, vernalization, and autonomous. The genes in these pathways function to perceive day length, internal rhythms, hormones, temperature, and developmental maturity, respectively. The photoperiod and circadian clock pathways work in tandem through the so-called external coincidence model, in which diurnal rhythms of circadian clock gene expression induce expression of downstream genes depending on the light cycle [10]. The outcomes of these pathways act on the FPIs to either enhance or suppress FPI and subsequent FMI gene expression.

In this study, an ortholog search using flowering time genes from other species was employed to identify putative flowering time genes in switchgrass. By comparing flowering-associated gene expression between upland and lowland ecotypes or between early- and late-flowering genotypes, this study aimed to elucidate differences due to ecotypeand flowering time-specific expression patterns. Results of this investigation will inform breeding efforts and/or advance genome engineering of switchgrass for improved biomass yield.

\section{Materials and Methods}

\section{Plant Material}

Switchgrass plants were grown at the University of Wisconsin Agricultural Research Station in Arlington, Wisconsin. The switchgrass genotypes used in this study were grown in a common garden that had been established 5 years prior to this study. Plants were established from vegetative ramets in May 2008 on a spacing of $0.9 \mathrm{~m}$ between adjacent plants. There were three clonal replicates of each of two upland genotypes, from the cultivar Cave-in-Rock, and two clonal replicates of each of the lowland genotypes, one from the cultivar High Tide and the other from the cultivar Kanlow (Table 1). The switchgrass genotypes used in this study broke dormancy in early May 2013, and tissue collection began shortly thereafter. In this study, flowering date was defined as the day of the year when at least $50 \%$ of the panicles on a plant reached R3, the fully expanded stage.

\section{Sample Collection}

Samples of three different tissues were taken at growth stages determined by leaf number, as defined in Moore et al. [11], and following a developmental series from emergence through anthesis. The tissues included the youngest emerging leaf blade, the entire blade of the second leaf at stage V2, a 2 $3-\mathrm{cm}$ section of the stem containing the shoot apical meristem (SAM), and, for stages after V2, the basal third of the blade of the fully collared leaf subtending the meristem (Fig. 1). For leaves at stages after V2, samples were collected twice: at the "early" stage, when the distance from the collar of the youngest fully collared leaf to the next emerging leaf tip was 15 $30 \mathrm{~cm}$ and at the "late" stage, when the distance from the collar to the next emerging leaf tip was double that of the "early" stage. "Early"-stage samples for V3 and V4 are denoted as V3.1 and V4.1, respectively. "Late"-stage samples for V3 and V4 are denoted as V3.2 and V4.2, respectively. An additional SAM-enriched sample at stage V5 was taken from each lowland genotype (5305-7 and 5300-8).

For each sample, tissue was pooled from four tillers per plant, with three replicate plants for each upland genotype (WS08-R3 and WS08-U1) and two for each lowland (5305- 
Table 1 Switchgrass genotype information, meristem transition stage, and flowering date

\begin{tabular}{lllll}
\hline Genotype & WS08-R3 & WS08-U1 & 5305-7 & 5300-8 \\
\hline Cultivar & Cave-in-Rock & Cave-in-Rock & High Tide & Kanlow \\
Ecotype & Upland & Upland & Lowland & Lowland \\
Flowering & Early & Early & Early & Late \\
Source & Illinois & Illinois & Maryland & Oklahoma \\
Replicates & 3 & 3 & 2 & 2 \\
SAM transition & June 7 $\left(158^{\mathrm{b}}\right)$ & June $7\left(158^{\mathrm{b}}\right)$ & June 20 $\left(171^{\mathrm{c}}\right)$ & June 28 $\left(179^{\mathrm{d}}\right)$ \\
Flowering date & August $8(220)$ & August $8(220)$ & August $15(227)$ & September 5 (248) \\
\hline a & & & & \\
${ }^{\mathrm{b}}$ Number in parentheses is day of year & & & \\
${ }^{\mathrm{c}}$ Stage is V3.1 & & & & \\
${ }^{\mathrm{d}}$ Stage is V4.1 & & & &
\end{tabular}

7 and 5300-8), as follows. For each replicate, after taking measurements and determining which tillers to collect tissue, four tillers were removed from the plant and all leaves below the uppermost collared leaf were removed from each tiller. Then, the basal third of the blade of the uppermost collared leaf from each tiller was collected and the pooled samples were immediately flash frozen in liquid nitrogen. Next, a 3$\mathrm{cm}$-long section (containing the SAM) of the internode adjacent to and above the uppermost collared leaf was dissected from each tiller, pooled, and flash frozen. Lastly, the emerging leaf tissue above the uppermost collared leaf was collected from each tiller, pooled, and flash frozen. Dissection of samples from the tillers was done rapidly, to limit the time between tiller harvest and sample freezing to less than $2 \mathrm{~min}$. All samples were kept in liquid nitrogen after flash freezing and during transport back to the laboratory in Madison, Wisconsin. Tissues were stored at $-80{ }^{\circ} \mathrm{C}$ until RNA extraction.

\section{Morphological Analysis of Shoot Apical Meristems}

For each stage at which samples were taken for possible RNA extraction, fresh tillers were also collected for analysis of the SAM morphology. At least three whole tillers at the corresponding stage were collected and kept on ice during transport back to the laboratory. Fresh tillers were stored for up to 3 days at $4{ }^{\circ} \mathrm{C}$, before microscopic analysis. Shoot apical meristems were dissected from surrounding sheath tissue, using a razor blade and an 18-gauge needle (Becton Dickinson, Franklin Lakes, NJ). Meristems were visualized under a Leica MZ16F stereomicroscope (Leica Microsystems, Switzerland) at various magnifications.

\section{RNA Extraction and Quantification}

RNA was extracted from selected samples taken at stages flanking and including meristem transition (Table 1), using the TRIzol (Life Technologies, Carlsbad, CA) method, following the manufacturer's protocol. Briefly, plant material was pulverized in liquid nitrogen, using a mortar and pestle to produce a fine powder, and approximately $100 \mathrm{mg}$ of powdered tissue was then used for RNA extraction in $1 \mathrm{~mL}$ of TRIzol. The RNA pellet was resuspended in $50 \mu \mathrm{L}$ RNAsefree water and the concentration checked on a NanoDrop, ND 1000 spectrophotometer (NanoDrop Technologies, Wilmington, DE). After determining the concentration, RNA integrity was checked via gel electrophoresis. RNA samples without degradation were purified using RNAeasy spin columns (Qiagen, Valencia, CA) and eluted in $30 \mu \mathrm{L}$ RNase-free water. Sample integrity and quality were further verified with an Agilent 2100 Bioanalyzer (Agilent Technologies, Germany), at the University of Wisconsin Biotechnology Center.

\section{RNA Sequencing and Sequence Processing}

Illumina TruSeq Stranded libraries were constructed and sequenced in paired end-mode at the DOE Joint Genome Institute (JGI) on an Illumina HiSeq 2000. One of the lowland samples, 5305-7 stage V4.2 SAM replicate 1, failed the final quality control and was not sequenced. However, the remaining 83 samples each produced an average of 25 million reads. Libraries from Cave-in-Rock were contaminated with switchgrass mosaic virus. To remove virus sequences, all libraries were aligned to the switchgrass mosaic virus genome (NC_015522.1) using TopHat v1.4.1 [12] with a minimum intron size of 5, maximum intron size of 5000, and the initial read mismatches $(-\mathrm{N})$ set to 7 ; all other parameters were set to default. All sequence reads were then cleaned using Cutadapt v1.4.1 [13] setting the quality score cutoff to $20(-\mathrm{q})$ and a minimum read length of 50. After cleaning, reads were assessed for quality using FastQC (http://www. bioinformatics.babraham.ac.uk/projects/fastqc/) and then trimmed to 150 nucleotides using FASTX TRIMMER from the FASTX-Toolkit v0.0.14 (http://hannonlab.cshl.edu/fastx toolkit/commandline.html). Cleaned reads were then aligned to the switchgrass reference genome sequence $(P$. virgatum 


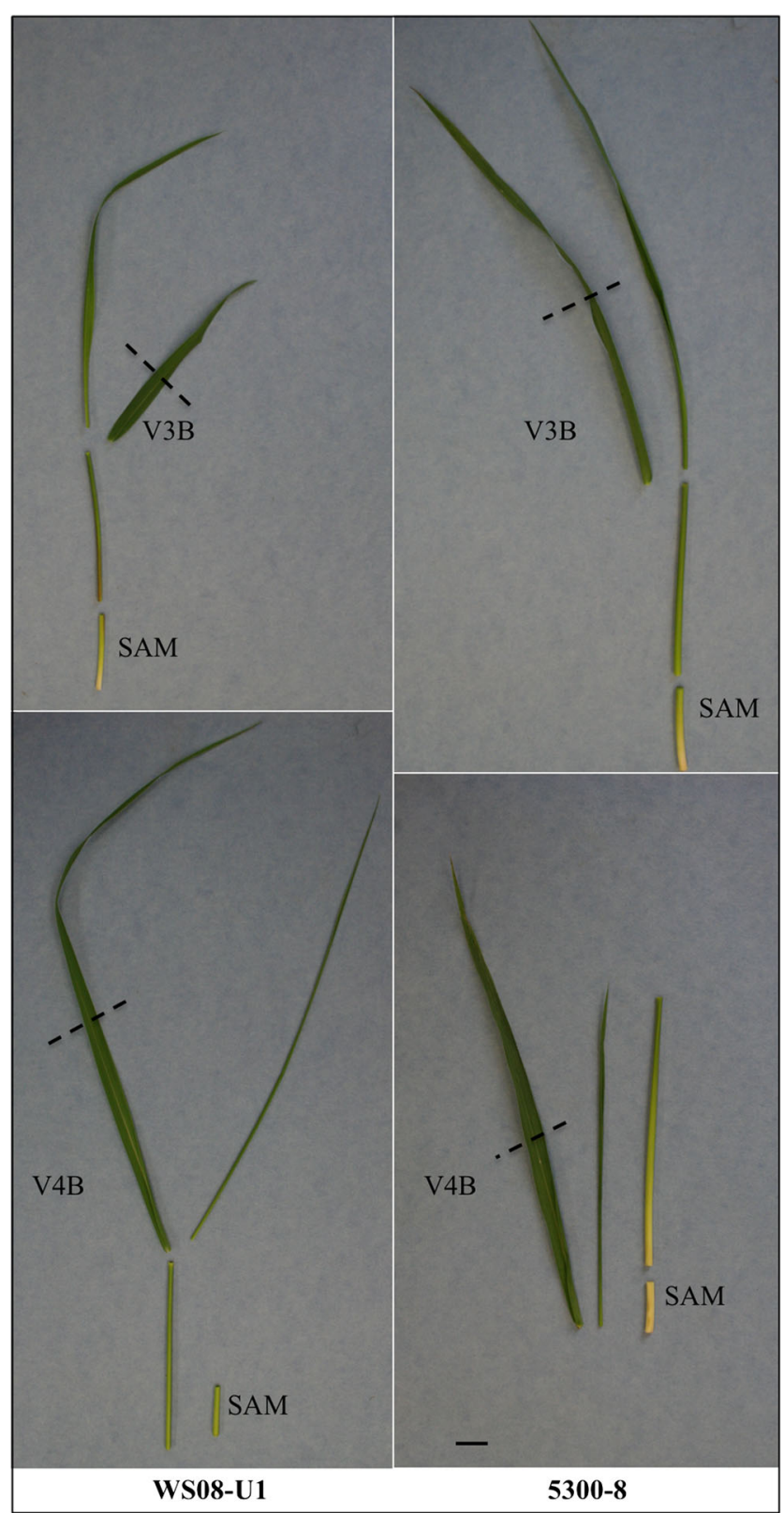

Fig. 1 Representative tissue types sampled. Shown are images of samples from two developmental stages. WS08-U1 is an early-flowering, upland genotype. 5300-8 is a late-flowering, lowland genotype. $S A M$ shoot apical meristem-enriched stem. $V 3 B$ (or $V 4 B$ ) basal third of leaf 3 (or 4). Dashed line marks the distal boundary of the sample collected. Bar $=2 \mathrm{~cm}$

v1.1, DOE-JGI, http://phytozome.jgi.doe.gov/) using TopHat v1.4.1 with a minimum intron size of $5 \mathrm{bp}$, a maximum intron size of $5000 \mathrm{bp}$, and segment length set to 25; all other parameters were set to default. Expression abundances (fragments per kilobase of gene model per million mapped reads (FPKM)) were generated using Cufflinks v1.3.0 [14] using both bias correction $(-b)$ and quantification against the reference transcript annotation $(-\mathrm{G})$, to generate an expression matrix of 98,005 genes across 83 samples (Online Resource $\mathrm{S} 1$ ). If the FPKM status for the gene was not "OK," the FPKM value was replaced with "NA" in the matrix. Raw sequence reads are available from the National Center for Biotechnology Information under project PRJNA361131.

\section{Expression Analysis}

Pearson's correlations between replicates indicated high reproducibility, with the lowest correlation of 0.9 for a single sample and most correlations in the 0.96-0.99 range (Online Resource S2). Of the 98,005 genes in the switchgrass reference genome (version 1.1), 81,430 had an FPKM $>0$ among the 83 samples sequenced. From the FPKM matrix, a mean expression matrix was calculated by averaging the FPKM values of the replicates (2 or 3 ) for each sample. Mean FPKM values for the genes with FPKM $>0$ in each of the 34 samples were used for principal component analysis (PCA) using the "prcomp" function in the $\mathrm{R}$ programming language [15].

For data exploration and differential expression analysis, the FPKM matrix was imported into ArrayStar software version 12.1 (DNASTAR, Madison, WI). For differential expression analysis, genotypes were grouped by ecotype: upland (WS08-R3 and WS08-U1) or lowland (5305-7 and 5300-8), and by flowering time: early- (WS08-R3, WS08-U1, and 5305-7) or late-flowering (5300-8). These groups are hereafter referred to as "comparison groups." Mean FPKM values for each tissue type of each comparison group were used for differential expression analyses.

\section{Identification of Flowering Time Orthologs}

Protein sequences of 93 known flowering time genes from Arabidopsis, maize, and/or rice were used to search for putative flowering time gene orthologs. OrthoMCL (v.1.4) analysis was performed using default parameters [16] with proteomes of six grass species including switchgrass (v1.1; phytozome.jgi.doe.gov), rice (v7; http://rice.plantbiology. msu.edu/), sorghum (v2.1; phytozome.jgi.doe.gov), $B$. distachyon (v1.2; phytozome.jgi.doe.gov), maize (v5b; maizesequence.org), and Setaria italica (v2.1; phytozome. jgi.doe.gov) with protein sequences of the 93 known flowering time genes; 448 gene orthologs from the six species including 101 from switchgrass were identified (Online Resource S3).

\section{Differential Expression Analysis of Flowering Time Orthologs}

For each tissue and developmental stage, the mean expression values (FPKM) of 101 switchgrass flowering time gene orthologs were compared between upland and lowland groups 
and between early- and late-flowering groups. Genes upregulated or downregulated by at least 1.7-fold ( $T$ test $p$ value $<0.05$ ) between the early- and late-flowering and/or upland and lowland comparison groups for the same tissue and stage and with a mean FPKM $>2$ (arbitrary FPKM threshold) in at least one of the comparison groups were deemed differentially expressed. The expression values of these differentially expressed genes were subsequently plotted for each comparison group and tissue type, along the entire developmental series, to compare expression patterns and classify genes as putative promoters or repressors of flowering.

\section{Results and Discussion}

\section{Dates of Flowering for Each Genotype}

In this study, flowering date was defined as the day of the year on which at least half of the tillers on a plant had reached R3, the stage at which panicles are fully expanded. The two upland genotypes, WS08-R3 and WS08-U1, flowered on August 8, or day 220 of the year. The early-flowering lowland genotype, 5305-7, flowered on August 15, or day 227 of the year. The late-flowering lowland genotype, 5300-8, flowered on September 5, or day 248 of the year (Table 1).

\section{Morphological Characterization of the SAM}

For the uplands, WS08-R3 and WS08-U1, which both flowered on the same date, the rachis, or first branch, of the reproductive meristem was detected in samples collected at the early V3 stage (V3.1), day 158 of the year. For the earlyflowering lowland genotype, 5305-7, which flowered a week after the uplands, the rachis meristem was detected at the late V3 stage (V3.2), or day 171 of the year. For the late-flowering lowland, 5300-8, which flowered 3 weeks after the uplands, the rachis meristem was detected in samples from the early V4 stage (V4.1), or day 179 of the year. Since the signal to transition from vegetative to reproductive meristem occurs before the change in SAM morphology is observed, the stage prior to the one in which the SAM transition was detected was used as the pre-transition, or vegetative SAM, sample for transcriptional analysis.

\section{Principal Component Analysis}

Out of 98,005 genes in the FPKM matrix, 16,575 had a FPKM value of zero in all samples analyzed. Therefore, 81,430 genes with FPKM $>0$ were used for PCA. Mean FPKM values for each of 34 samples were used for PCA. Combined, principal components 1 and 2 (PC1 and PC2) accounted for approximately $42 \%$ of the total variation within the FPKM matrix.
Fig. 2 Principal component analysis (PCA) of FPKM expression values for 34 sample types sequenced from four genotypes. The entire FPKM matrix contains expression data for 98,005 genes, of which 16,575 had an FPKM value of zero in all samples sequenced. Therefore, 81,430 genes had FPKM values $>0$ within the sample set and were used for the PCA. Principal components 1 and 2 (PC1 and PC2) account for $\sim 42 \%$ of the total variation within the FPKM matrix. PC1 clearly accounts for tissue variation and PC2 accounts for genotype, ecotype, or flowering time variation. Each point is the eigenvalue calculated for each sample, from mean global expression values from three or two replicates for upland and lowland genotypes, respectively

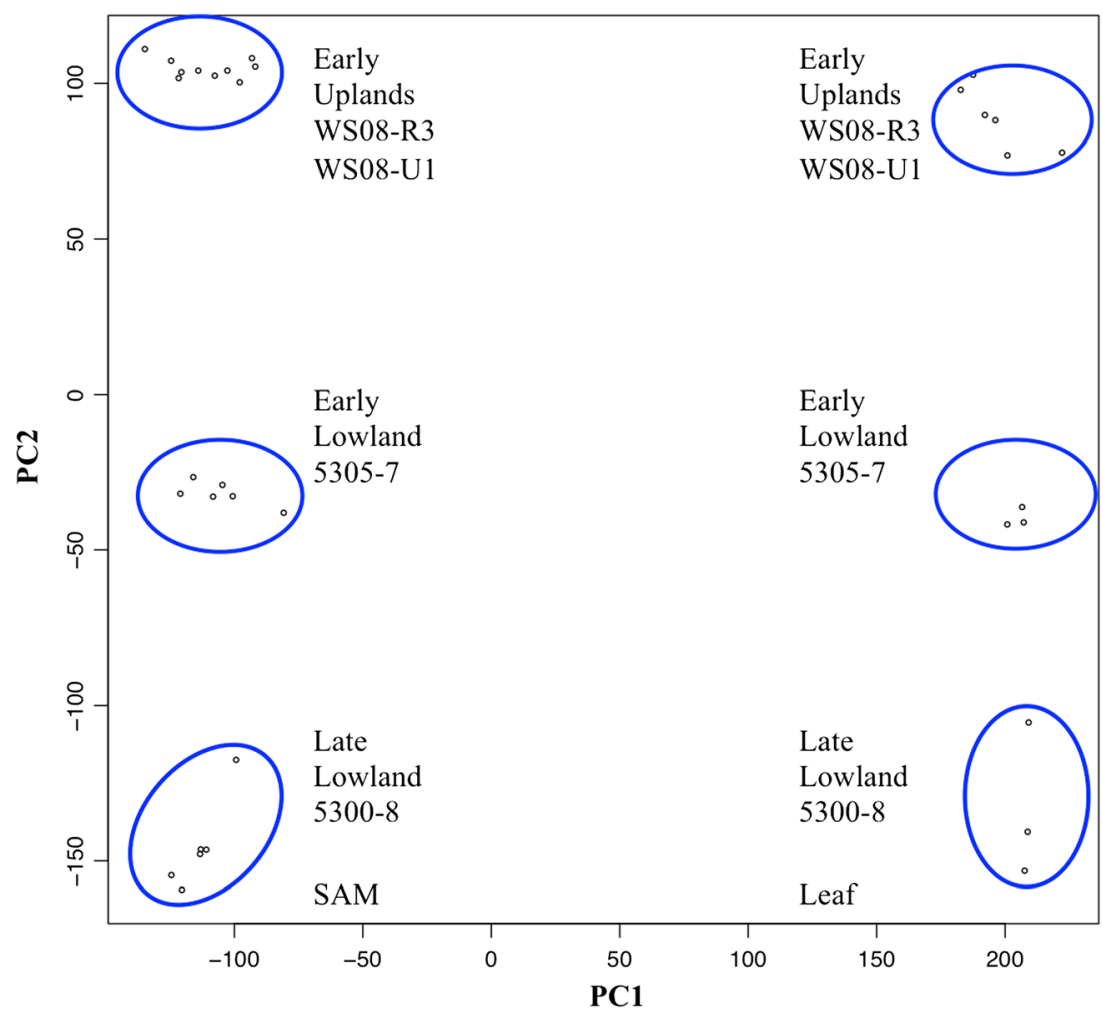


PC1 clearly accounted for tissue variation and PC2 accounted for genotype, ecotype, and/or flowering time variation (Fig. 2), indicating differential expression between these categories.

\section{Differential Expression of Known Flowering Time Gene Orthologs}

For 23 of the 93 known flowering-associated genes that had been identified in Arabidopsis, rice, and/or maize, no orthologs were identified in the switchgrass reference genome version 1.1; however, this could be due to an incomplete version of the genome. For the 70 known floweringassociated genes with identified switchgrass orthologs, 101, in total, were found. Of the 101 switchgrass orthologs, 23 had mean FPKM values of less than two (FPKM threshold) in all tissues across the four comparison groups (Online Resource S4). Therefore, 78 switchgrass orthologs met the FPKM threshold for at least one comparison group and were used for differential expression analyses. The arbitrary cutoff of FPKM >2 removed some known key players in the flowering pathway that may be expressed at low levels (e.g., FT/TSF); however, we were most interested in genes that were expressed early on, before meristem transition, that play roles upstream of FPI/FMI components.

Hierarchical clustering analysis of the 78 switchgrass orthologs with mean FPKM values greater than two for at least one comparison group revealed coexpressed genes and, for the most part, identical expression patterns between groups

Fig. 3 Heat map of expression of 78 switchgrass flowering time candidate gene orthologs in leaves, after hierarchical clustering. Expression values are the $\log _{2}$-transformed mean FPKM for each comparison group, upland (WS08-R3 and WS08-U1) and lowland (5305-7 and 5300-8). Headings indicate comparison group and sample. The gene tree is shown on the left and gene IDs on the right. Arrowheads mark genes that were classified as promoters or repressors of flowering. The color-scale legend at the bottom shows the range of expression as $\log _{2}$-transformed FPKM values

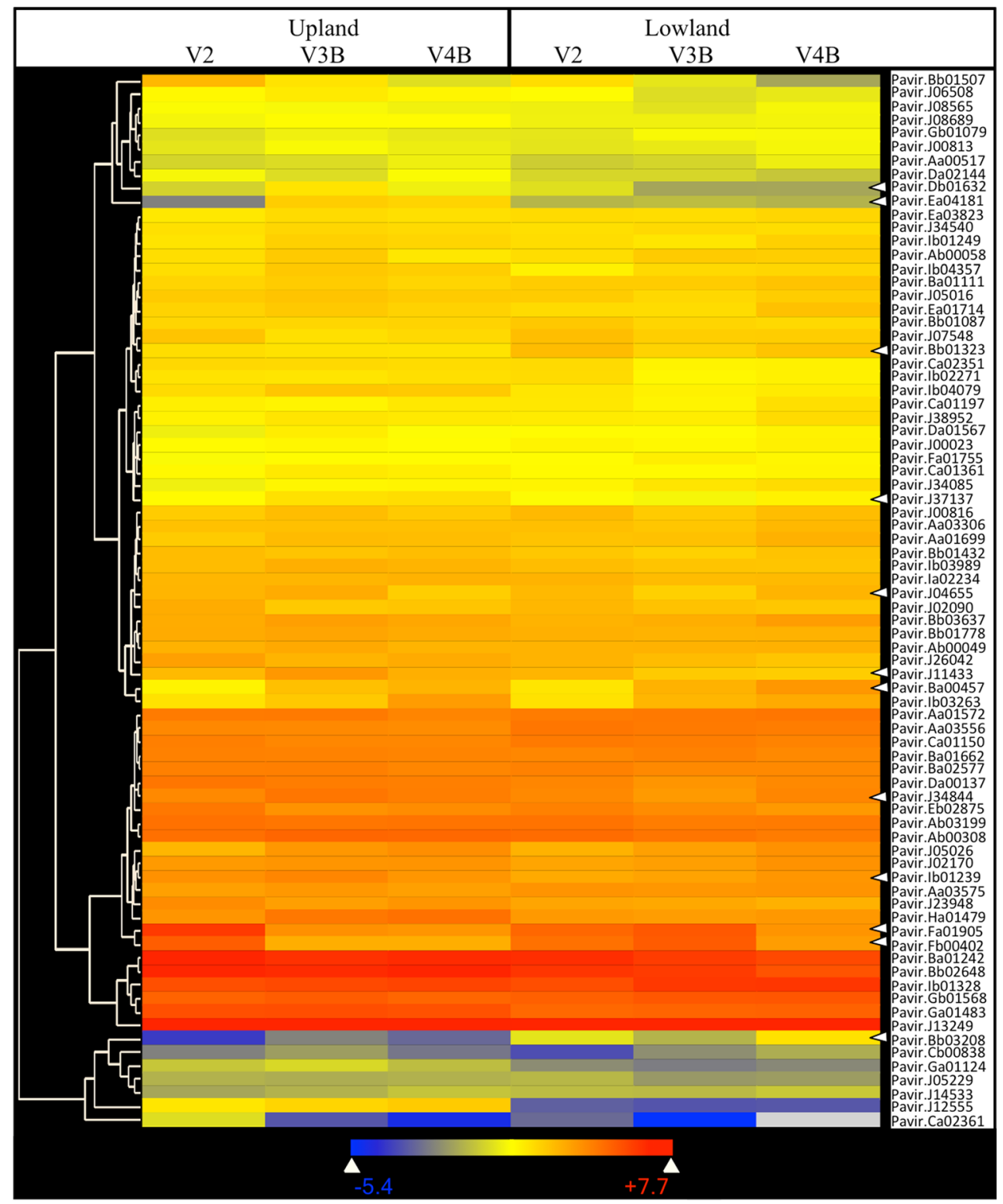


with differing flowering times. However, for a number of orthologs, differential expression was detected between comparison groups (Figs. 3, 4, 5, and 6).

The developmental stage of SAM transition, as determined by microscopy, informed the selection of tissue stages to sequence. The stages analyzed included the stage of SAM transition and stages flanking the transition, with the expectation that genes involved in flowering time would have differential expression from one transition flanking stage to the other. In addition, genes necessary for flowering time differences between comparison groups were expected to have differential expression between those groups. Based on the stage or stages at which genes had differential expression between comparison groups, the genes could be classified as putative promoters or repressors of flowering or having an unknown role.
Putative promoters of flowering had higher expression in early stages (V2, V3) in upland or early-flowering groups than in lowland or late-flowering groups, respectively. Conversely, putative repressors of flowering had higher expression in early stages (V2, V3) in lowland or late-flowering, compared to upland or early-flowering groups, respectively. Genes that fit the criteria for being a putative promoter or repressor are presented here.

\section{Promoters of Flowering}

Switchgrass orthologs of seven known flowering time genes were classified as putative floral promoters (Table 2). Five of these genes fall into the photoperiod/circadian clock pathway, one in the autonomous, and one in the vernalization pathway.

Fig. 4 Heat map of expression of 78 switchgrass flowering time candidate gene orthologs in SAM-enriched tissue, after hierarchical clustering. Expression values are the $\log _{2}$-transformed mean FPKM for each comparison group, upland (WS08-R3 and WS08-U1) and lowland (5305-7 and 5300-8). Headings indicate comparison group and sample. The gene tree is shown on the left and gene IDs on the right. Arrowheads mark genes that were classified as promoters or repressors of flowering. The color-scale legend at the bottom shows the range of expression as $\log _{2}$-transformed FPKM values

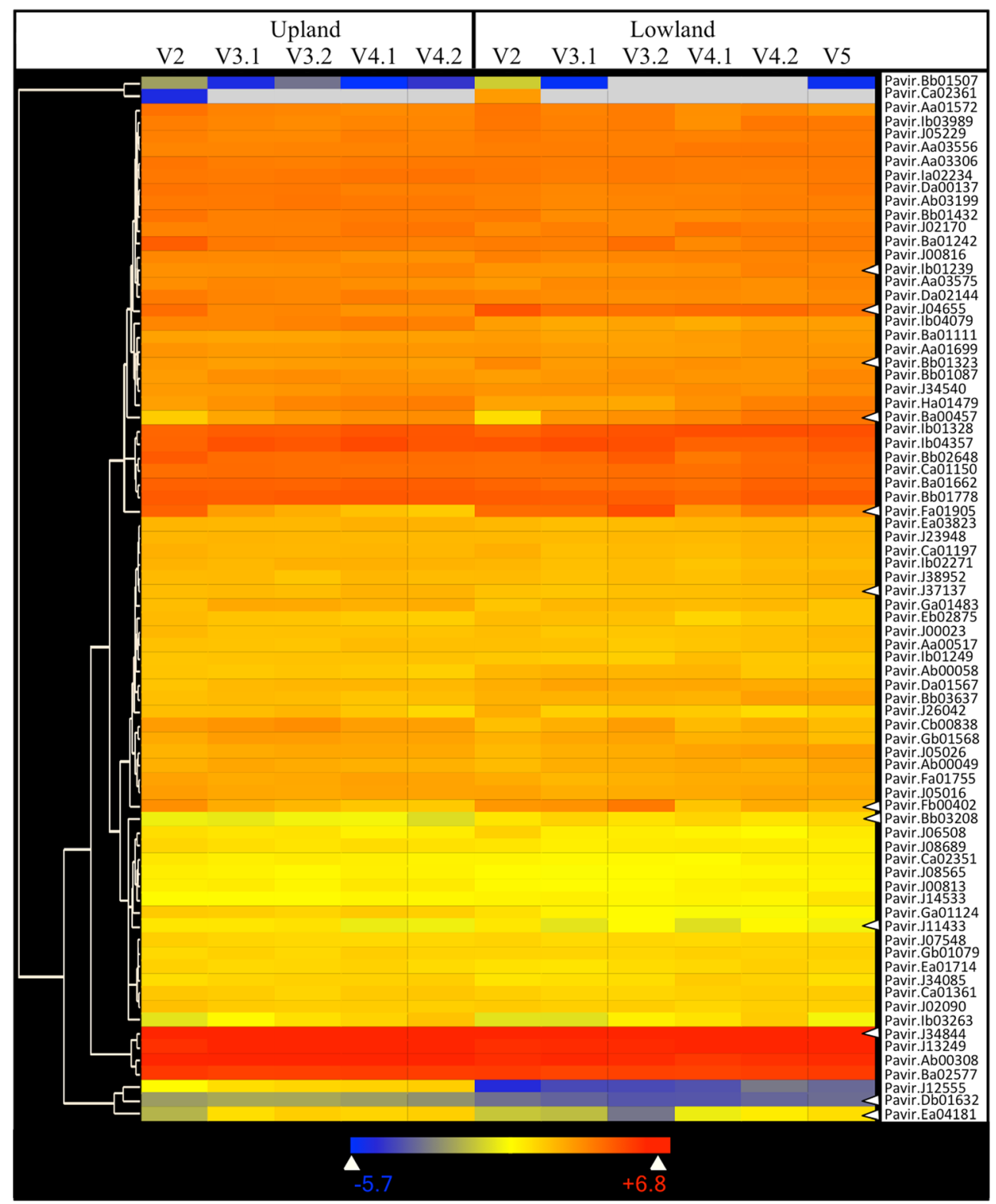


Fig. 5 Heat map of expression of 78 switchgrass flowering time candidate gene orthologs in leaves, after hierarchical clustering. Expression values are the $\log _{2}$-transformed mean FPKM for each comparison group, early (WS08-R3, WS08U1, and 5305-7) and late (5300$8)$. Headings indicate comparison group and sample. The gene tree is shown on the left and gene IDs on the right. Arrowheads mark genes that were classified as promoters or repressors of flowering. The color-scale legend at the bottom shows the range of expression as $\log _{2}$-transformed FPKM values

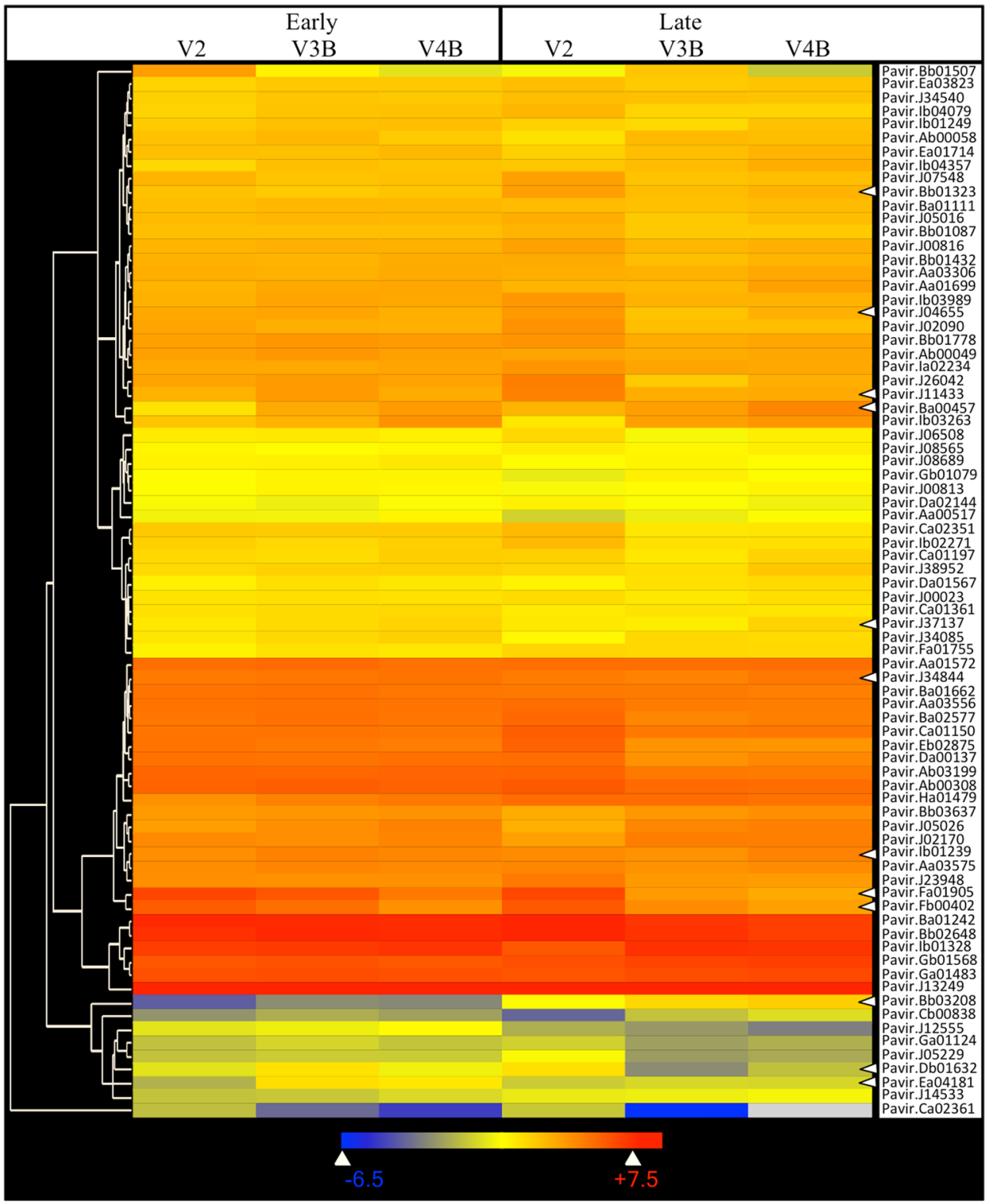

One striking example of a putative floral promoter is the gene Pavir.Ea04181, an ortholog of the Arabidopsis gene, LUX ARRYTMO (LUX) [17]. In leaves of lowland and late-flowering genotypes (5305-7 and 5300-8), Pavir.Ea04181 had little to no expression throughout the three developmental stages analyzed, while the expression level in early-flowering, upland genotypes (WS08-R3 and WS08-U1) spiked at the stage of SAM transition as determined by microscopy (Fig. 7a). LUX, a Myb domain transcription factor, has been shown in Arabidopsis to be essential for producing circadian rhythms. Specifically, the proposed function of LUX is upregulation of the photoperiod floral activation pathway and acts indirectly to upregulate the photoperiod/circadian clock gene CONSTANS (CO) via GIGANTEA (GI) [18], thereby activating FPIs and FMIs in the subsequent SAM transition [17]. In the SAM of upland genotypes (WS08-R3 and WS08-U1), Pavir.Ea04181 expression increased from V2 stage to V3.1, where it plateaued. In lowland genotypes (5305-7 and 5300-8), Pavir.Ea04181 expression began at the V3.1 stage, increasing sharply to the level of uplands at stage V4.2 (Fig. 7a). Of note, in Fig. 7a, are the overlapping expression patterns of the lowland (5305-7 and 5300-8) and lateflowering (5300-8) groups, suggesting an ecotype-specific expression pattern.

\section{Repressors of Flowering}

Switchgrass orthologs of five known flowering time genes were classified as putative floral repressors (Table 3). Two of 
Fig. 6 Heat map of expression of 78 switchgrass flowering time candidate gene orthologs in SAM-enriched tissue, after hierarchical clustering. Expression values are the $\log _{2}$-transformed mean FPKM for each comparison group, early (WS08-R3, WS08U1, and 5305-7) and late (53008). Headings indicate comparison group and sample. The gene tree is shown on the left and gene IDs on the right. Arrowheads mark genes that were classified as promoters or repressors of flowering. The color-scale legend at the bottom shows the range of expression as $\log _{2}$-transformed FPKM values

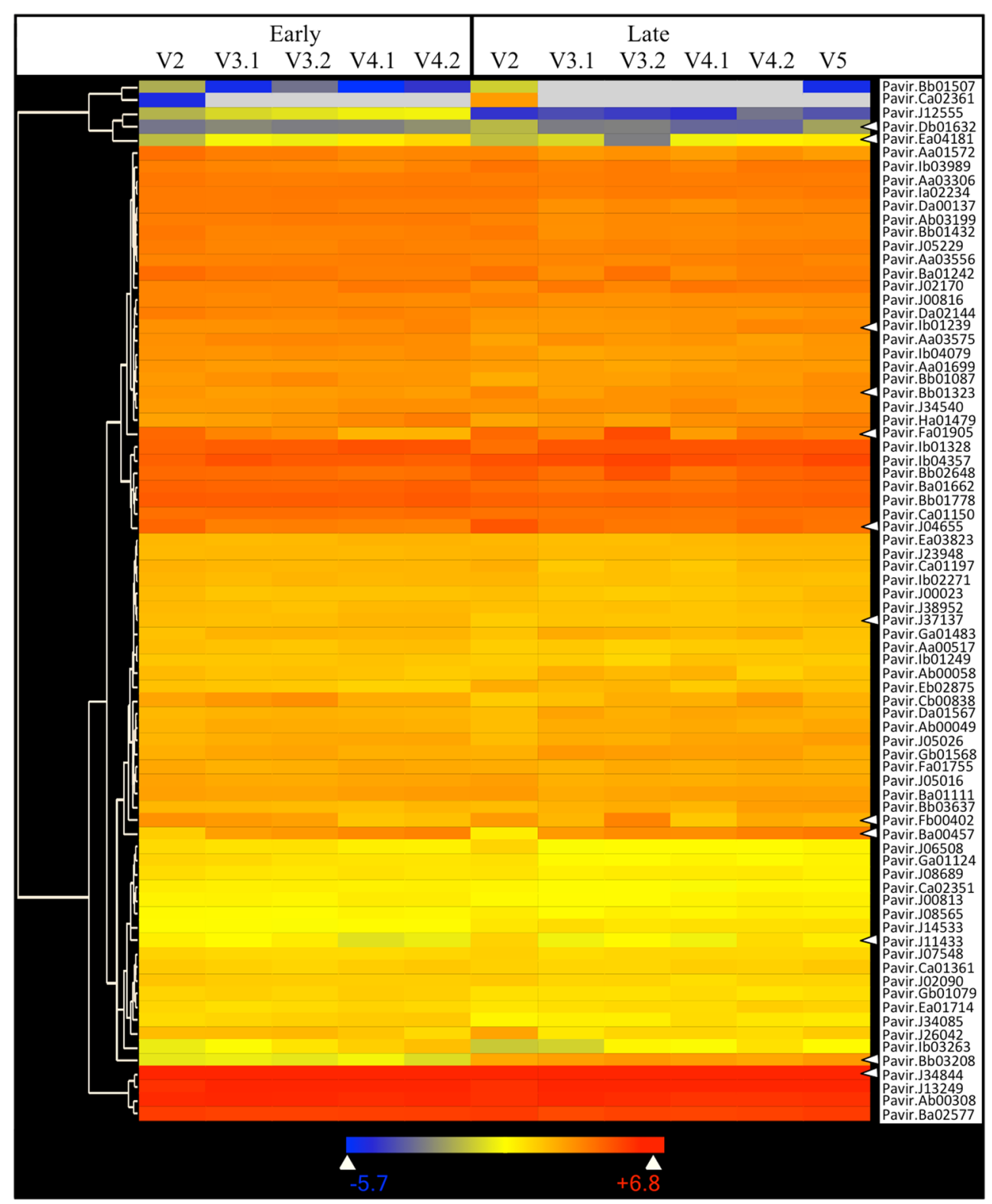

these genes fall into the category of FPI or FMI genes, two in the autonomous, and one in the photoperiod/clock pathway.

An example of a putative floral repressor that shows dramatic differential expression between flowering time groups is Pavir.Bb03208, an ortholog of the Arabidopsis gene, BRI1ESSENTIAL 1 (BES1) [19]. In leaves of the upland and earlyflowering genotypes (WS08-R3, WS08-U1, and 5305-7), Pavir.Bb03208 had little to no expression (FPKM <1) over the three developmental stages analyzed, while in the lateflowering, lowland genotype (5300-8), Pavir.Bb03208 transcript was present in gradually increasing levels through the series of the three leaf stages (Fig. 7b). Since BES1 in Arabidopsis represses FPI gene expression [20, 21], the lack of BES1 expression in the early-flowering genotypes suggests a de-repression of floral activators, indicative of the transition to reproductive meristem growth.

In the SAM of the late-flowering, lowland genotype (53008), Pavir.Bb03208 expression is more than 6-fold higher than in the early-flowering genotypes (WS08-R3, WS08-U1, and 5305-7), suggesting a role in suppression of floral activator expression in the SAM. Note that the expression patterns of Pavir.Bb03208 in the upland (WS08-R3 and WS08-U1) and early-flowering (WS08-R3, WS08-U1, and 5305-7) comparison groups are nearly identical, while the expression pattern of the late-flowering genotype (5300-8) differs from the lowland group (5305-7 and 5300-8) pattern. This suggests that the expression of Pavir.Bb03208 in the SAM is flowering timespecific. 
Table 2 Putative floral promoters identified through differential expression analysis of switchgrass flowering time orthologs

\begin{tabular}{|c|c|c|c|c|c|c|}
\hline Locus & Gene ID & Late leaf ${ }^{\mathrm{a}}$ & Late $\mathrm{SAM}^{\mathrm{b}}$ & Low leaf ${ }^{c}$ & Low SAM $^{\mathrm{d}}$ & Pathway \\
\hline Pavir.J34844 & MSI4/FVE & & & $\mathrm{V} 3(0.5)$ & & Autonomous \\
\hline Pavir.Db01632 & CONSTANS & $\begin{array}{l}\mathrm{V} 2(3) ; \mathrm{V} 3(0.1) ; \mathrm{V} 4 \\
\quad(0.4)\end{array}$ & & $\begin{array}{l}\mathrm{V} 3(0.1) ; \mathrm{V} 4 \\
\quad(0.3)\end{array}$ & & Photoperiod/clock \\
\hline Pavir.J04655 & PHYA & V3 $(0.5)$ & & $\begin{array}{l}\mathrm{V} 3(0.5) \text {; V4 } \\
\quad(1.6)\end{array}$ & V2, V3.1 (1.5); V4.1,V4.2 (1.9) & Photoperiod/clock \\
\hline Pavir.Fa01905 & CCA1-like & & $\begin{array}{l}\mathrm{V} 3.2(2.8) ; \mathrm{V} 4.2 \\
\quad(3)\end{array}$ & V2 (0.4) & $\begin{array}{l}\text { V3.1 (2.4); V3.2 (5.1); V4.1 (1.9); V4.2 } \\
\quad(3.9)\end{array}$ & Photoperiod/clock \\
\hline Pavir.Ib01239 & PHYB & & & V2, V3 (0.6) & & Photoperiod/clock \\
\hline Pavir.Ea04181 & LUX & & & V3, V4 (0.1) & $\begin{array}{l}\text { V3.1 (0.2); V3.2 (0.04); V4.1 (0.4); V4.2 } \\
\quad(0.6)\end{array}$ & Photoperiod/clock \\
\hline Pavir.J37137 & EFS & & & V3 $(0.5)$ & & Vernalization \\
\hline
\end{tabular}

${ }^{a}$ Relative expression in leaf in late- versus early-flowering groups. Leaf stage indicated. Fold change in parentheses

${ }^{\mathrm{b}}$ Relative expression in SAM in late- versus early-flowering groups. SAM stage indicated. Fold change in parentheses

${ }^{\mathrm{c}}$ Relative expression in leaf in lowland versus upland groups. Leaf stage indicated. Fold change in parentheses

${ }^{\mathrm{d}}$ Relative expression in SAM in lowland versus upland groups. SAM stage indicated. Fold change in parentheses

\section{Conclusions}

In this study, flowering time differences, measured as heading date, among upland and lowland genotypes were mirrored in the SAM transition dates, suggesting that the date of transition from vegetative to reproductive meristem defines the time of flowering. Orthologs of known flowering time genes had either ecotype- or flowering time-specific patterns, allowing for the possible separation of flowering time traits from determinants of other ecotype-specific traits in breeding or transgenic manipulation efforts. Lastly, known flowering time gene orthologs from multiple pathways, but mainly the photoperiod/clock and autonomous pathways, were differentially expressed between genotypes with differing flowering times, indicating conservation of these pathways, at least in part, across species.

At the convergence of the flowering time pathway, found in both monocots and dicots, is the FPI and mobile signal called "florigen," which is translocated from the leaf through the phloem to the SAM [22]. The specific gene that encodes the florigen protein was discovered in Arabidopsis and named FLOWERING LOCUS T (FT) [23-25]. Genes with FT-like functions have been identified in rice [26], maize [7, 27], and Brachypodium [5]. Even with conserved function, not all flowering time gene orthologs function in the same capacity across plant species. In rice, for example, there are two florigens, one that promotes flowering in short days only and the other acting in long days only [28]. For switchgrass, investigations into the flowering time pathway have also revealed complexities. A recent report on control of floral transition in switchgrass analyzed the expression of a switchgrass FT ortholog [29]. The authors found that, in greenhouse conditions, FT transcript increased from the V3 to E3 stage and suggested that the floral transition occurred between stages
E1 to E3. Interestingly, both of the switchgrass FT orthologs identified here were not expressed, with mean comparison group FPKM values below 2 across all tissues, despite having observed morphological changes in the SAM that indicated the occurrence of the transition from vegetative to reproductive growth. The possible reasons for the discrepancy in FT expression between the Niu et al. [29] study and this are 3fold: (1) there may be other FT-like genes acting as FPIs that were not identified in the ortholog search, (2) the expression of the switchgrass FT ortholog may have been spatially restricted to the middle or top section of the leaf blade or the leaf sheath tissues which were not included in this study, and (3) there may be differences in FT expression among different genotypes, as the ones surveyed here are different from the single lowland variety used in the Niu et al. [29] study. Not only could FT expression patterns differ between genotypes, but the numerous FT-like genes in switchgrass may work in concert, each having partial roles in control of flowering. In addition, in a recent switchgrass flowering time association study, a single nucleotide polymorphism (SNP) in an FT-like gene was found to be associated with early flowering [30]. However, the early flowering allele was evident in only northern populations, leading the authors to suggest possible subfunctionalization of this FT homolog to play a role in cold hardiness [30].

The gene candidates evaluated in this study provide insight into the flowering time pathway in switchgrass. By surveying multiple genotypes from two ecotypes with different flowering times, gene candidates could be classified as either putative promoters or repressors of flowering. In addition, due to one lowland genotype having early flowering, expression patterns could be parsed out as ecotype- or flowering timespecific, thereby being able to dissect pathways shared between ecotypes. Orthologs of known floral genes, particularly 


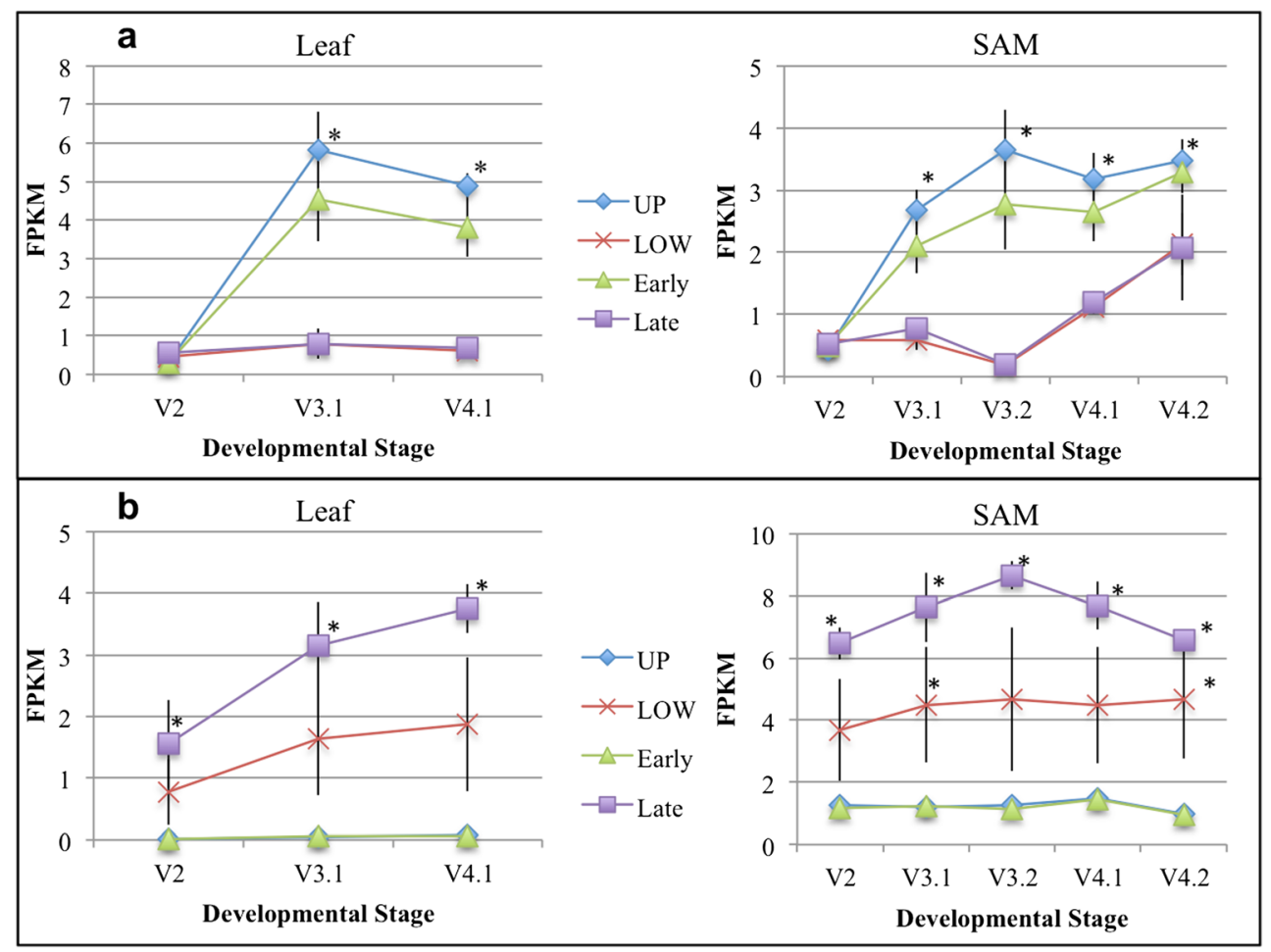

Fig. 7 Switchgrass orthologs of two known flowering time genes are differentially expressed between genotypes from two ecotypes. a Expression levels in FPKM of Pavir.Ea04181 in the leaf (left) and SAM-enriched tissue (right) along a developmental series from the second through the fourth leaf stage (V2-V4). Pavir.Ea04181 is an ortholog of the Arabidopsis gene, LUX ARRHYTHMO (LUX), a promoter of flowering. b Expression patterns, as in a, of Pavir.Bb03208 in the leaf (left) and SAM-enriched tissue (right). Pavir.Bb03208 is an ortholog of the Arabidopsis gene, BRI1-EMS-SUPRESSOR 1 (BES1), a repressor of

from the photoperiod/circadian clock pathway, were expressed differentially in ecotype- and flowering timespecific manners, suggesting that parts of these pathways may have different expression patterns between ecotypes, but play no role in determining flowering time. Several novel genes that encode protein domains like those found in known flowering time genes were identified, such as Myb and zincfinger transcription factors. These novel gene candidates all showed flowering time-specific expression patterns, indicating a shared pathway across ecotypes. It remains to be seen whether these conserved genes also share conserved functions or have evolved divergent functions as with the rice FT orthologs.

Switchgrass is photoperiodic, but the day length requirements are variable between cultivars and have been shown to correlate with latitude of origin, with southern-derived cultivars having a short-day requirement and northern cultivars responding to long days or being photoperiod-insensitive [31]. High night temperatures have been suggested to restrict flowering in some cultivars of switchgrass [32]; therefore, cool nights may possibly induce flowering, or, at least, permit it. Flowering in switchgrass is influenced by many factors, flowering. Each data point is the mean FPKM value of the respective grouping. Groupings are: UP, both upland genotypes (WS08-R3 and WS08-U1); LOW, both lowland genotypes (5305-7 and 5300-8); Early, both uplands and the early-flowering lowland genotype (WS08-R3, WS08-U1, and 5305-7); and Late, the single late-flowering lowland genotype (5300-8). Significant differences between early-/late-flowering or upland/lowland, as determined by a $T$ test with $p$ value $<0.05$, are denoted by an asterisk. Error bars are $+/-$ standard error

both endogenous and exogenous. Some components of the photoperiod and autonomous pathways have been identified in switchgrass [33]; however, investigations in sorghum, another high biomass crop, have yielded more pieces of the flowering pathway in grasses. Three well-characterized maturity loci in sorghum, Ma1, Ma3, and Ma6, harbor floral repressors that confer photoperiod sensitivity [34]. Ma1 corresponds to SbPRR37, the ortholog of Arabidopsis PRR7 [35]; PHYB is the gene at the Ma3 locus [36]; and SbGhd7, the ortholog of rice Ghd7, was identified as Ma6 [37]. Work by Wolabu et al. [38] has shown that sorghum has 19 genes that encode a phosphatidylethanolamine-binding protein (PEBP) domain - the characteristic domain of FT and TERMINAL FLOWER LIKE 1 (TFL1), florigen and antiflorigen, respectively. Three of the 19 PEBP-containing genes have been shown to act as functional florigens, responding to different cues, and two are TFL1-like [38]. The model of multiple florigens is supported by previous work in rice, with its two florigens activated by different photoperiods. Switchgrass, with over 40 FT-like genes [30], could possibly also have multiple florigens that are activated by different photoperiods or environmental conditions. 
Table 3 Putative floral repressors identified through differential expression analysis of switchgrass flowering time orthologs

\begin{tabular}{|c|c|c|c|c|c|c|}
\hline Locus & Gene ID & Late leaf ${ }^{\mathrm{a}}$ & Late $\mathrm{SAM}^{\mathrm{b}}$ & Low leaf ${ }^{\mathrm{c}}$ & Low SAM $^{\mathrm{d}}$ & Pathway \\
\hline Pavir.Bb01323 & FCA-like & V2 (2) & & $\begin{array}{l}\text { V2 (1.8); } \\
\quad \text { V3 (1.7) }\end{array}$ & & Autonomous \\
\hline Pavir.J11433 & RAP2-7 & V2 (2.4) & V4.2 (2.3) & & & FPI/FMI \\
\hline Pavir.Ba00457 & AGL8-like & V2 (2.3) & & & & FPI/FMI \\
\hline Pavir.Bb03208 & BES1 & $\begin{array}{l}\text { V2 (20); V3 } \\
\text { (21); V4 (26) }\end{array}$ & $\begin{array}{l}\text { V2 (5.6); V3.1 (6.2); V3.2 (7.7); } \\
\text { V4.1 (5.4); V4.2 (6.9) }\end{array}$ & $\begin{array}{l}\text { V2 (20); } \\
\quad \text { V4 (26) }\end{array}$ & $\begin{array}{l}\text { V2 (2); V3.1 (3.1); V3.2 (2); } \\
\text { V4.1 (2.5); V4.2 (4) }\end{array}$ & Autonomous \\
\hline Pavir.Fb00402 & CCA1-like & & & V3 (4.4) & $\mathrm{V} 3.2(2.8)$ & Photoperiod/clock \\
\hline
\end{tabular}

${ }^{a}$ Relative expression in leaf in late- versus early-flowering groups. Leaf stage indicated. Fold change in parentheses

${ }^{\mathrm{b}}$ Relative expression in SAM in late- versus early-flowering groups. SAM stage indicated. Fold change in parentheses

${ }^{\mathrm{c}}$ Relative expression in leaf in lowland versus upland groups. Leaf stage indicated. Fold change in parentheses

${ }^{\mathrm{d}}$ Relative expression in SAM in lowland versus upland groups. SAM stage indicated. Fold change in parentheses

Subsequent steps to confirm and analyze function of these candidate genes could be carried out initially in the model grass Brachypodium, by using readily available mutant knockout lines and/or through genetic transformations. However, as Brachypodium is a C3 annual grass, it may not serve as the best system for functional analyses of switchgrass genes. Setaria viridis, a C4 grass closely related to switchgrass has been developed for floral dip transformation [39] and, therefore, may be a promising system for determining the functions of switchgrass candidate genes. Recently, a virus-based technique for delivering florigens to various plant systems has been described [40]. This technique allows for transient expression of florigens in species that are difficult to stably transform. The main benefit of virus-induced flowering is the ability to stage plants with highly divergent flowering times for crossing; however, this could be an efficient way to assess the function of florigens (or anti-florigens) directly in switchgrass. In addition, concurrent flowering time QTL mapping studies using populations generated from upland/lowland switchgrass hybrids are expected to support the findings in this study. The identification of components of the switchgrass flowering time pathway that are potential promoters or repressors will enable future breeding and/or transgenic work to improve switchgrass biomass yield through modification of flowering time. For example, CONSTANS, the central component of the photoperiod response pathway in Arabidopsis, is upregulated in early-flowering genotypes, compared to late-flowering genotypes. One could possibly alter the promoter region of CONSTANS, through gene editing, to suppress its expression in upland varieties to delay flowering. In addition, upstream of CONSTANS, in the photoperiod/ circadian clock-coupled pathway, a number of genes in this study showed differential expression between genotypes of differing flowering times. One example detailed earlier is LUX, but the phytochromes, PHYA and PHYB, that entrain the circadian clock [10] also showed differential expression. It could be that the late-flowering switchgrass genotypes in this study may have variation in the clock-entraining mechanisms that result in differential photoperiod sensitivity. Functional analyses of the differentially expressed orthologs presented here are, therefore, warranted.

Acknowledgments This work was funded by the US Department of Energy (DOE) Great Lakes Bioenergy Research Center (DOE BER Office of Science DE-FC02-07ER64494). We thank the US DOE Joint Genome Institute for sequencing work and advance access to the v.1.1 Panicum virgatum genome. The US DOE Joint Genome Institute work was funded by the Office of Science of the US DOE under contract no. DE-AC02-05CH11231.

\section{Compliance With Ethical Standards}

Funding This work was funded by the US Department of Energy (DOE) Great Lakes Bioenergy Research Center (DOE BER Office of Science DE-FC02-07ER64494) and by the US DOE Office of Science DE-AC02-05CH11231.

Conflict of Interest The authors declare that they have no conflict of interest.

Open Access This article is distributed under the terms of the Creative Commons Attribution 4.0 International License (http:// creativecommons.org/licenses/by/4.0/), which permits unrestricted use, distribution, and reproduction in any medium, provided you give appropriate credit to the original author(s) and the source, provide a link to the Creative Commons license, and indicate if changes were made.

\section{References}

1. Casler MD (2012) Switchgrass breeding, genetics, and genomics. In: Monti A (ed) Switchgrass: a valuable biomass crop for energy. Springer, London, pp 29-53

2. Casler MD, Tobias CM, Kaeppler SM, Buell CR, Wang Z-Y, Cao P, Schmutz J, Ronald P (2011) The switchgrass genome: tools and 
strategies. Plant Genome 4(3):273-282. doi:10.3835/ plantgenome2011.10.0026

3. Taliaferro CM (2002) Breeding and selection of new switchgrass varieties for increased biomass production. Oak Ridge National Laboratory, Oak Ridge, Tennessee

4. Vogel KP, Brejda JJ, Walters DT, Buxton DR (2002) Switchgrass biomass production in the midwest USA: harvest and nitrogen management. Agron J 94:413-420

5. Higgins JA, Bailey PC, Laurie DA (2010) Comparative genomics of flowering time pathways using Brachypodium distachyon as a model for the temperate grasses. PLoS One 5(4):e10065. doi:10. 1371/journal.pone.0010065

6. Amasino R (2010) Seasonal and developmental timing of flowering. Plant J 61(6):1001-1013. doi:10.1111/j.1365-313X. 2010.04148.x

7. Lazakis CM, Coneva V, Colasanti J (2011) ZCN8 encodes a potential orthologue of Arabidopsis FT florigen that integrates both endogenous and photoperiod flowering signals in maize. J Exp Bot 62(14):4833-4842. doi:10.1093/jxb/ err129

8. Tamaki S, Matsuo S, Wong HL, Yokoi S, Shimamoto K (2007) $\mathrm{Hd} 3$ a protein is a mobile flowering signal in rice. Science 316(5827):1033-1036. doi:10.1126/science.1141753

9. Woods DP, Ream TS, Minevich G, Hobert O, Amasino RM (2014) PHYTOCHROME C is an essential light receptor for photoperiodic flowering in the temperate grass, Brachypodium distachyon. Genetics 198(1):397-408. doi:10. 1534/genetics.114.166785

10. Johansson M, Staiger D (2015) Time to flower: interplay between photoperiod and the circadian clock. J Exp Bot 66(3):719-730. doi: 10.1093/jxb/eru441

11. Moore K, Moser L, Vogel K, Waller S, Johnson B, Pedersen J (1991) Describing and quantifying growth stages of perennial forage grasses. Agron J 83:1073-1077

12. Trapnell C, Pachter L, Salzberg SL (2009) TopHat: discovering splice junctions with RNA-Seq. Bioinformatics 25(9):1105-1111. doi:10.1093/bioinformatics/btp120

13. Martin M (2011) Cutadapt removes adapter sequences from highthroughput sequencing reads. EMBnetjournal 17(1):10-12. doi:10. 14806/ej.17.1.200

14. Trapnell C, Williams BA, Pertea G, Mortazavi A, Kwan G, van Baren MJ, Salzberg SL, Wold BJ, Pachter L (2010) Transcript assembly and quantification by RNA-Seq reveals unannotated transcripts and isoform switching during cell differentiation. Nat Biotechnol 28(5):511-515. doi:10.1038/nbt.1621

15. R Core Team (2014) R: a language and environment for statistical computing. R Foundation for Statistical Computing, Vienna, Austria

16. Li L, Stoeckert CJ, Roos DS (2003) OrthoMCL: identification of ortholog groups for eukaryotic genomes. Genome Res 13(9):21782189. doi: $10.1101 /$ gr. 1224503

17. Hazen SP, Schultz TF, Pruneda-Paz JL, Borevitz JO, Ecker JR, Kay SA (2005) LUX ARRHYTHMO encodes a Myb domain protein essential for circadian rhythms. Proc Natl Acad Sci U S A 102(29): 10387-10392. doi:10.1073/pnas.0503029102

18. Song YH, Estrada DA, Johnson RS, Kim SK, Lee SY, MacCoss MJ, Imaizumi T (2014) Distinct roles of FKF1, Gigantea, and Zeitlupe proteins in the regulation of Constans stability in Arabidopsis photoperiodic flowering. Proc Natl Acad Sci U S A 111(49):17672-17677. doi:10. 1073/pnas.1415375111

19. Yin Y, Vafeados D, Tao Y, Yoshida S, Asami T, Chory J (2005) A new class of transcription factors mediates brassinosteroidregulated gene expression in Arabidopsis. Cell 120(2):249-259. doi:10.1016/j.cell.2004.11.044

20. Jiang J, Zhang C, Wang X (2015) A recently evolved isoform of the transcription factor BES1 promotes brassinosteroid signaling and development in Arabidopsis thaliana. Plant Cell 27(2):361-374. doi: $10.1105 /$ tpc. 114.133678

21. Clouse SD (2008) The molecular intersection of brassinosteroid-regulated growth and flowering in Arabidopsis. Proc Natl Acad Sci U S A 105(21):73457346. doi:10.1073/pnas.0803552105

22. Zeevaart JA (2008) Leaf-produced floral signals. Curr Opin Plant Biol 11(5):541-547. doi:10.1016/j.pbi.2008.06.009

23. Kardailsky I, Shukla VK, Ahn JH, Dagenais N, Christensen SK, Nguyen JT, Chory J, Harrison MJ, Weigel D (1999) Activation tagging of the floral inducer FT. Science 286(5446): 1962-1965

24. Kobayashi Y, Kaya H, Goto K, Iwabuchi M, Araki T (1999) A pair of related genes with antagonistic roles in mediating flowering signals. Science 286(5446):1960-1962

25. Corbesier L, Vincent C, Jang S, Fornara F, Fan Q, Searle I, Giakountis A, Farrona S, Gissot L, Turnbull C, Coupland G (2007) FT protein movement contributes to long-distance signaling in floral induction of Arabidopsis. Science 316(5827):1030-1033. doi:10.1126/science. 1141752

26. Komiya R, Ikegami A, Tamaki S, Yokoi S, Shimamoto K (2008) $\mathrm{Hd} 3 \mathrm{a}$ and RFT1 are essential for flowering in rice. Development 135(4):767-774. doi:10.1242/dev.008631

27. Meng X, Muszynski MG, Danilevskaya ON (2011) The FT-like ZCN8 gene functions as a floral activator and is involved in photoperiod sensitivity in maize. Plant Cell 23(3):942-960. doi:10.1105/ tpc. 110.081406

28. Tsuji H, Taoka K, Shimamoto K (2011) Regulation of flowering in rice: two florigen genes, a complex gene network, and natural variation. Curr Opin Plant Biol 14(1):45-52. doi:10.1016/j.pbi.2010. 08.016

29. Niu L, Fu C, Lin H, Wolabu TW, Wu Y, Wang ZY, Tadege M (2016) Control of floral transition in the bioenergy crop switchgrass. Plant Cell Environ. doi:10.1111/pce.12769

30. Grabowski PP, Evans J, Daum C, Deshpande S, Barry KW, Kennedy M, Ramstein G, Kaeppler SM, Buell CR, Jiang Y, Casler MD (2017) Genome-wide associations with flowering time in switchgrass using exome-capture sequencing data. New Phytol 213 (1):154-169. doi:10.1111/nph.14101

31. McMillan C (1959) The role of ecotypic variation in the distribution of the central grassland of North America. Ecol Monogr 29(4):286-308

32. McMillan C (1965) Ecotypic differentiation within four North American prairie grasses. II. Behavioral variation within transplanted community fractions. Am J Bot 52(1):55-65

33. Nuñez F, Yamada T (2017) Molecular regulation of flowering time in grasses. Agronomy 7(1):17

34. Wolabu TW, Tadege M (2016) Photoperiod response and floral transition in sorghum. Plant Signal Behav 11(12):e1261232. doi: 10.1080/15592324.2016.1261232

35. Murphy RL, Klein RR, Morishige DT, Brady JA, Rooney WL, Miller FR, Dugas DV, Klein PE, Mullet JE (2011) Coincident light and clock regulation of pseudoresponse regulator protein 37 (PRR37) controls photoperiodic flowering in sorghum. Proc Natl Acad Sci U S A 108(39):1646916474. doi:10.1073/pnas.1106212108

36. Childs KL, Miller FR, Cordonnier-Pratt MM, Pratt LH, Morgan PW, Mullet JE (1997) The sorghum photoperiod sensitivity gene, Ma3, encodes a phytochrome B. Plant Physiol 113(2):611-619

37. Murphy RL, Morishige DT, Brady JA, Rooney WL, Yang S, Klein PE, Mullet JE (2014) Ghd7 (Ma6) represses sorghum flowering in long days: Ghd7 alleles enhance biomass accumulation and grain production. Plant Genome 7(2). doi:10.3835/plantgenome2013.11. 0040 
38. Wolabu TW, Zhang F, Niu L, Kalve S, Bhatnagar-Mathur P, Muszynski MG, Tadege M (2016) Three FLOWERING LOCUS T-like genes function as potential florigens and mediate photoperiod response in sorghum. New Phytol 210(3):946-959. doi:10.1111/ nph.13834

39. Martins PK, Nakayama TJ, Ribeiro AP, Cunha, BADBd, Nepomuceno, AL, Harmon, FG, Kobayashi, AK, Molinari, HBC
(2015) Setaria viridis floral-dip: a simple and rapid Agrobacterium-mediated transformation method. Biotechnology Reports 6:61-63

40. McGarry RC, Klocko AL, Pang M, Strauss SH, Ayre BG (2017) Virus-induced flowering: an application of reproductive biology to benefit plant research and breeding. Plant Physiol 173(1):47-55. doi:10.1104/pp.16.01336 